\title{
Quadratic Forms and Nonlinear Non-Resonant Singular Second Order Boundary Value Problems of Limit Circle Type
}

\author{
R. P. Agarwal, D. O'Regan and V. Lakshmikantham
}

Abstract. New existence results are presented for non-resonant second order singular boundary value problems

$$
\left.\begin{array}{l}
\frac{1}{p(t)}\left(p(t) y^{\prime}(t)\right)^{\prime}+\tau(t) y(t)=\lambda f(t, y(t)) \quad \text { a.e. on }[0,1] \\
\lim _{t \rightarrow 0^{+}} p(t) y^{\prime}(t)=y(1)=0
\end{array}\right\}
$$

where one of the endpoints is regular and the other may be singular or of limit circle type.

Keywords: Singular and non-resonant problems, points of limit circle type, existence criteria for solutions

AMS subject classification: $34 \mathrm{~B} 15$

\section{Introduction}

In this paper we develop an existence theory for

$$
\frac{1}{p(t)}\left(p(t) y^{\prime}(t)\right)^{\prime}+\tau(t) y(t)=\lambda f(t, y(t)) \quad \text { a.e. on }[0,1]
$$

which makes use of the relationship between the asymptotic behavior of the non-linearity $\frac{f(t, y)}{y}$ and the spectrum of the differential operator. In particular, we examine the nonresonant second order singular boundary value problem

$$
\left.\begin{array}{l}
\frac{1}{p(t)}\left(p(t) y^{\prime}(t)\right)^{\prime}+\tau(t) y(t)=\lambda f(t, y(t)) \text { a.e. on }[0,1] \\
\lim _{t \rightarrow 0^{+}} p(t) y^{\prime}(t)=y(1)=0
\end{array}\right\} \text {. }
$$

R. P. Agarwal: Nat. Univ. Singapore, Dept. Math., 10 Kent Ridge Crescent, Singapore 119260 D. O'Regan: Nat. Univ. of Ireland, Dept. Math., Galway, Ireland

V. Lakshmikantham: Florida Inst. Techn., Dept. Math. Sci., Melbourne, Florida 32901, USA matravip@nus.edu.sg; donal.oregan@ucg.ie; gsac@zach.fit.edu 
Throughout $p \in C[0,1] \cap C^{1}(0,1)$ together with $p>0$ on $(0,1), \tau$ is measurable with $\tau>0$ a.e. on $[0,1]$ and $\int_{0}^{1} p(x) \tau(x) d x<\infty$, and $\lambda \in \mathbb{R}$ is some parameter. We do not assume $\int_{0}^{1} \frac{d s}{p(s)}<\infty$ but rather $\int_{0}^{1} \frac{1}{p(s)}\left(\int_{0}^{s} p(x) \tau(x) d x\right)^{\frac{1}{2}} d s<\infty$. As a result for the eigenvalue problem

$$
\left.\begin{array}{l}
L u=\lambda u \text { a.e. on }[0,1] \\
\lim _{t \rightarrow 0^{+}} p(t) u^{\prime}(t)=u(1)=0
\end{array}\right\}
$$

where $L u=-\frac{1}{p q}\left(p u^{\prime}\right)^{\prime}$, one of the endpoints, $t=1$, will be regular and the other, $t=0$, may be singular or of limit circle type $[6,7]$. For nonlinear non-resonant problems of limit circle type only a handful of papers have appeared in the literature (see $[1,3,6]$ ). All other papers, to our knowledge, concerning nonlinear non-resonant problems discuss the case when $t=0$ and $t=1$ are regular points (see $[2,4,5,7]$ and the references therein). In [6], Fonda and Mawhin presented a technique for discussing non-resonant problems (i.e. (1.1) with $p \equiv 1$ ) based on quadratic forms. We will use part of this technique in this paper. However, as we will see, many extra steps will be needed to discuss non-resonant problems when one of the endpoints is of limit circle type.

For notational purposes let $w$ be a weight function. By $L_{w}^{2}[0,1]$ we mean the space of functions $u$ such that $\int_{0}^{1} w(t)|u(t)|^{2} d t<\infty$ (also, if $u \in L_{w}^{2}[0,1]$, we define $\left.\|u\|_{w}=\left(\int_{0}^{1} w(t)|u(t)|^{2} d t\right)^{\frac{1}{2}}\right)$. Let $A C[0,1]$ be the space of functions which are absolutely continuous on $[0,1]$.

The following well known existence principle $[6,7]$ (which is a special case of the Leray-Schauder continuation theorem), due to O'Regan, will be needed in Section 2.

Theorem 1.1. Suppose the following conditions are satisfied:

(i) $p \in C[0,1] \cap C^{1}(0,1)$ with $p>0$ on $(0,1)$.

(ii) $\tau \in L_{p}^{1}[0,1]$ with $\tau>0$ a.e. on $[0,1]$.

(iii) $\int_{0}^{1} \frac{1}{p(s)}\left(\int_{0}^{s} p(x) \tau(x) d x\right)^{1 / 2} d s<\infty$.

(iv) $f:[0,1] \times \mathbb{R} \rightarrow \mathbb{R}$ is a Carathéodory function, i.e.

(i) $t \mapsto f(t, y)$ is measurable for all $y \in \mathbb{R}$

(ii) $y \mapsto f(t, y)$ is continuous for a.e. $t \in[0,1]$.

(v) $\frac{f(t, y(t))}{\tau(t)} \in L_{p \tau}^{2}[0,1]$ whenever $y \in L_{p \tau}^{2}[0,1]$.

In addition, assume that problem $\left(P_{0}\right)$ has only the trivial solution. Further, suppose there is a constant $M_{0}$, independent of $\lambda$, with

$$
\|y\|_{p \tau}=\left(\int_{0}^{1} p(t) \tau(t)|y(t)|^{2} d t\right)^{\frac{1}{2}} \neq M_{0}
$$

for any solution $y$ (here $y \in L_{p \tau}^{2}[0,1]$ with $y \in C(0,1] \cap C^{1}(0,1)$ and $\left.p y^{\prime} \in A C[0,1]\right)$ to problem $\left(P_{\lambda}\right)$, for each $\lambda \in(0,1)$. Then problem $\left(P_{1}\right)$ has at least one solution. 
Finally, we remark that problems of type $\left(P_{\lambda}\right)$ occur in many applications in the physical sciences, for example in radially symmetric nonlinear diffusion in the $n$-dimensional sphere we have $p(t)=t^{n-1}$; these problems involve a homogeneous Neumann condition at zero, i.e. $\lim _{t \rightarrow 0^{+}} t^{n-1} u^{\prime}(t)=0$. Another example is the Poisson-Boltzmann equation

$$
\left.\begin{array}{rlrl}
y^{\prime \prime}+\frac{\alpha}{t} y^{\prime} & =f(t, y) & & (0<t<1) \\
y^{\prime}\left(0^{+}\right) & =y(1)=0 & & (\alpha \geq 1)
\end{array}\right\}
$$

which occurs in the theory of thermal explosions and in the theory of electrohydrodynamics. The results related to problem (1.2) in the literature $[1,3]$ usually consider the situation when inf $\frac{\partial f}{\partial y}$ and $\sup \frac{\partial f}{\partial y}$ are bounded and satisfy a "non-resonant" condition. In this paper we improve the above existence result (in fact, in our theory the existence of $\frac{\partial f}{\partial y}$ is not assumed).

We also note that the results in [6] are a special case of Theorems 2.1 and 2.2 in this paper (see the special example after the proof of Theorem 2.1).

\section{Non-resonance type problems}

In this section we present two existence results for singular boundary value problem $\left(P_{1}\right)$. Conditions (i) - (v) of Theorem 1.1 will be assumed throughout this section. Notice condition (iii) implies (see [7]) $\int_{0}^{1} p(x) \tau(x)\left(\int_{x}^{1} \frac{d s}{p(s)}\right)^{2} d x<\infty$.

Our first result establishes existence if a certain integral inequality is satisfied.

Theorem 2.1. Suppose conditions (i) - (v) of Theorem 1.1 hold and suppose problem $\left(P_{0}\right)$ has only the trivial solution. In addition, assume $f$ has the decomposition

$$
f(t, u)=g_{1}(t, u) u+g_{2}(t, u)+h(t, u)
$$

where $g_{1}, g_{2}, h:[0,1] \times \mathbb{R} \rightarrow \mathbb{R}$ are Carathéodory functions and the following conditions are satisfied:

(i) $u g_{2}(t, u) \geq 0$ for a.e. $t \in[0,1]$ and $u \in \mathbb{R}$.

(ii) $\exists \tau_{1} \in C[0,1]$ with $\tau_{1}(t) \tau(t) \leq g_{1}(t, u) \leq 0$ for a.e. $t \in[0,1]$ and $u \in \mathbb{R}$.

(iii) $|h(t, u)| \leq \phi_{1}(t)+\phi_{2}(t)|u|^{\gamma}$ for a.e. $t \in[0,1]$, with $0 \leq \gamma<1$.

(iv) $\int_{0}^{1} p(t) \phi_{1}(t)\left(\int_{t}^{1} \frac{d s}{p(s)}\right)^{1 / 2} d t<\infty$ and $\int_{0}^{1} p(t) \phi_{2}(t)\left(\int_{t}^{1} \frac{d s}{p(s)}\right)^{(\gamma+1) / 2} d t<\infty$.

(v) $\int_{0}^{1}\left[p\left(u^{\prime}\right)^{2}-\left(\tau-\tau_{1} \tau\right) p u^{2}\right] d t>0$ for any $0 \neq u \in K^{\star}$

where

$$
K^{\star}=\left\{\begin{array}{l|l}
w:[0,1] \rightarrow \mathbb{R} & \begin{array}{l}
w \in L_{p \tau}^{2}[0,1] \text { with } w \in C(0,1] \\
w^{\prime} \in L_{p}^{2}[0,1] \text { and } w(1)=0
\end{array}
\end{array}\right\} .
$$

Then problem $\left(P_{1}\right)$ has a solution $y \in L_{p \tau}^{2}[0,1]$ with $y \in C(0,1] \cap C^{1}(0,1)$ and $p y^{\prime} \in$ $A C[0,1]$. 
Proof. We first show that there exists $\varepsilon>0$ with

$$
\int_{0}^{1}\left[p\left(y^{\prime}\right)^{2}-\left(\tau-\tau_{1} \tau\right) p y^{2}\right] d t \geq \varepsilon\left(\|y\|_{p \tau}^{2}+\left\|y^{\prime}\right\|_{p}^{2}\right)
$$

for any $y \in K^{\star}$. If this is not the case, then there exists a sequence $\left\{y_{n}\right\} \subset K^{\star}$ with

$$
\begin{aligned}
& \left\|y_{n}\right\|_{p \tau}^{2}+\left\|y_{n}^{\prime}\right\|_{p}^{2}=1 \\
& \int_{0}^{1}\left[p\left(y_{n}^{\prime}\right)^{2}-\left(\tau-\tau_{1} \tau\right) p y_{n}^{2}\right] d t \rightarrow 0 \quad \text { as } n \rightarrow \infty
\end{aligned}
$$

The Riesz compactness criteria together with a standard result in functional analysis (if $E$ is a reflexive Banach space, then any norm bounded sequence in $E$ has a weakly convergent subsequence) implies that there is a subsequence $S$ of integers with

$$
y_{n} \rightarrow y \text { in } L_{p \tau}^{2}[0,1] \quad \text { and } \quad y_{n}^{\prime} \rightarrow y^{\prime} \text { in } L_{p}^{2}[0,1]
$$

as $n \rightarrow \infty$ in $S$ where $\rightarrow$ denotes weak convergence.

Note $\left\{y_{n}\right\}$ is bounded in $L_{p \tau}^{2}[0,1]$ (see $(2.2)$ ) and, for $r>0$, Hölder's inequality yields

$$
\begin{aligned}
\int_{0}^{1} p(t) \tau(t)\left|y_{n}(t+r)-y_{n}(t)\right|^{2} d t & =\int_{0}^{1} p \tau \int_{t}^{t+r} y_{n}^{\prime}(s) d s \quad d t \\
& \leq\left\|y_{n}^{\prime}\right\|_{p}^{2} \int_{0}^{1} p \tau \int_{t}^{t+r} \frac{d s}{p(s)} d t \\
& \leq \int_{0}^{1} p \tau \int_{t}^{1} \frac{d s}{p(s)} d t-\int_{0}^{1} p \tau \int_{t+r}^{1} \frac{d s}{p(s)} d t \\
& \rightarrow 0 \text { as } r \rightarrow 0^{+}
\end{aligned}
$$

by the Lebesgue dominated convergence theorem and assumption (iii) of Theorem 1.1. Thus $\left\{y_{n}\right\}$ is relatively compact in $L_{p \tau}^{2}[0,1]$.

Next, a standard result in functional analysis [7] yields

$$
\int_{0}^{1} p\left[y^{\prime}\right]^{2} d t \leq \liminf \int_{0}^{1} p\left[y_{n}^{\prime}\right]^{2} d t
$$

Now $(2.3)-(2.5)$ and the fact that $\liminf \left[s_{n}+t_{n}\right] \geq \liminf s_{n}+\liminf t_{n}$ for sequences $\left\{s_{n}\right\}$ and $\left\{t_{n}\right\}$ imply

$$
\int_{0}^{1}\left[p\left(y^{\prime}\right)^{2}-\left(\tau-\tau_{1} \tau\right) p y^{2}\right] d t \leq 0
$$

since

$$
\liminf \int_{0}^{1}\left(\tau-\tau_{1} \tau\right) p y_{n}^{2} d t=\int_{0}^{1}\left(\tau-\tau_{1} \tau\right) p y^{2} d t
$$


Note $y(1)=0$ since in fact $y_{n} \rightarrow y$ in $C[\varepsilon, 1](\varepsilon>0)$ by the Arzela-Ascoli theorem. By assumption (v) we have $y \equiv 0$. However,

$$
\begin{aligned}
\left\|y_{n}\right\|_{p \tau}^{2}+\left\|y_{n}^{\prime}\right\|_{p}^{2}= & \int_{0}^{1} p \tau y_{n}^{2} d t+\int_{0}^{1}\left(\tau-\tau_{1} \tau\right) p y_{n}^{2} d t \\
& +\int_{0}^{1}\left[p\left(y_{n}^{\prime}\right)^{2}-\left(\tau-\tau_{1} \tau\right) p y_{n}^{2}\right] d t \\
\rightarrow & \text { as } n \rightarrow \infty \text { in } S
\end{aligned}
$$

which is impossible. Thus (2.1) holds for some $\varepsilon>0$.

Let $y$ be a solution to problem $\left(P_{\lambda}\right)$ for some $0<\lambda<1$. Note, in particular, $y \in K^{\star}$. Multiply the differential equation by $y$ and integrate from 0 to 1 to obtain

$$
\int_{0}^{1}\left[p\left(y^{\prime}\right)^{2}-\tau p y^{2}\right] d t=-\lambda \int_{0}^{1} p y^{2} g_{1}(t, y) d t-\lambda \int_{0}^{1} p y g_{2}(t, y) d t-\lambda \int_{0}^{1} p y h(t, y) d t
$$

and so (use assumptions (i) - (ii))

$$
\int_{0}^{1}\left[p\left(y^{\prime}\right)^{2}-\left(\tau-\tau_{1} \tau\right) p y^{2}\right] d t \leq \int_{0}^{1} p|y h(t, y)| d t .
$$

This together with assumption (iii) and (2.1) imply that there exists $\varepsilon>0$ (fix it) with

$$
\varepsilon\left(\|y\|_{p \tau}^{2}+\left\|y^{\prime}\right\|_{p}^{2}\right) \leq \int_{0}^{1} p \phi_{1}|y| d t+\int_{0}^{1} p \phi_{2}|y|^{\gamma+1} d t .
$$

Since $y(1)=0$, we have from Hölder's inequality

$$
|y(t)|=\left|\int_{t}^{1} y^{\prime}(s) d s\right| \leq\left\|y^{\prime}\right\|_{p}\left(\int_{t}^{1} \frac{d s}{p(s)}\right)^{\frac{1}{2}}
$$

for $t \in(0,1)$, and so

$$
\varepsilon\left(\|y\|_{p \tau}^{2}+\left\|y^{\prime}\right\|_{p}^{2}\right) \leq K_{0}\left\|y^{\prime}\right\|_{p}+K_{1}\left\|y^{\prime}\right\|_{p}^{\gamma+1}
$$

where

$$
K_{0}=\int_{0}^{1} p(t) \phi_{1}(t)\left(\int_{t}^{1} \frac{d s}{p(s)}\right)^{\frac{1}{2}} d t \quad \text { and } \quad K_{1}=\int_{0}^{1} p(t) \phi_{2}(t)\left(\int_{t}^{1} \frac{d s}{p(s)}\right)^{\frac{\gamma+1}{2}} d t .
$$

Now (2.7) guarantees that there is a constant $M>0$, independent of $\lambda$, with $\left\|y^{\prime}\right\|_{p} \leq M$. This together with (2.7) guarantees the existence of a constant $M_{0}>0$, independent of $\lambda$, with $\|y\|_{p \tau} \leq M_{0}$. The result now follows from Theorem 1.1 
We now discuss briefly assumption (v) of Theorem 2.1. Inequalities of this type play a major role in the literature of calculus of variation. We illustrate the ideas involved with a simple example. Consider the problem

$$
\left.\begin{array}{rl}
\frac{1}{p}\left(p y^{\prime}\right)^{\prime}+\mu q y & =f(t, y) \text { a.e. on }[0,1] \\
\lim _{t \rightarrow 0^{+}} p(t) y^{\prime}(t) & =y(1)=0
\end{array}\right\}
$$

with $q \in L_{p}^{1}[0,1], q>0$ a.e. on $[0,1]$, and

$$
\mu\left(1-\tau_{1}(t)\right)<\lambda_{0} \text { for } t \in[0,1]
$$

$\lambda_{0}$ being the first eigenvalue of problem (1.1) with $L u=-\frac{1}{p q}\left(p u^{\prime}\right)^{\prime}$. Let also assumptions (i), (iii) - (v) of Theorem 1.1 and assumptions (i) - (iv) of Theorem 2.1 hold, with $\tau(t)=$ $\mu q(t))$. Recall (see [7: Chapter 11], limit circle case) that $L$ has a countable number of real eigenvalues $\lambda_{i}>0$ (arranged so that $\lambda_{0}<\lambda_{1}<\lambda_{2}<\ldots$ ) with corresponding (orthonormal) eigenfunctions $\psi_{i}$. The set $\left\{\psi_{i}\right\}$ form a basis of $L_{p q}^{2}[0,1]$, and so for any $u \in K^{\star}$ we have

$$
u(t)=\sum_{i=0}^{\infty} \eta_{i} \psi_{i}(t), \quad \eta_{i}=\left\langle u, \psi_{i}\right\rangle_{p q}
$$

where $\langle u, v\rangle_{p q}=\int_{0}^{1} p q u \bar{v} d t$.

We claim that problem (2.8) has at least one solution. This follows immediately from Theorem 2.1 once we show its condition (v) is satisfied. First notice from (2.9) (note $\tau_{1} \in C[0,1]$ ) that there exists $\delta>0$ with $\mu\left(1-\tau_{1}(t)\right) \leq \lambda_{0}-\delta$ for $t \in[0,1]$. Now for $u \in K^{\star}$ we have

$$
\begin{aligned}
\int_{0}^{1}\left[p\left(u^{\prime}\right)^{2}-\left(\tau-\tau_{1} \tau\right) p u^{2}\right] d t & \geq \int_{0}^{1}\left[p\left(u^{\prime}\right)^{2}-\left(\lambda_{0}-\delta\right) p q u^{2}\right] d t \\
& =\sum_{i=0}^{\infty} \eta_{i}^{2}\left[\lambda_{i}-\left(\lambda_{0}-\delta\right)\right] \int_{0}^{1} p q \psi_{i}^{2} d t
\end{aligned}
$$

since $\left(p \psi_{i}^{\prime}\right)^{\prime}+\lambda_{i} p q \psi_{i}=0$ a.e. on $[0,1]$ and $\lim _{t \rightarrow 0^{+}} p(t) \psi_{i}(t)=\psi_{i}(1)=0$. Consequently,

$$
\int_{0}^{1}\left[p\left(u^{\prime}\right)^{2}-\left(\tau-\tau_{1} \tau\right) p u^{2}\right] d t \geq \delta \sum_{i=0}^{\infty} \eta_{i}^{2} \int_{0}^{1} p q \psi_{i}^{2} d t=\delta \int_{0}^{1} p q|u|^{2} d t>0
$$

for $u \neq 0$. Thus condition (v) of Theorem 2.1 holds, so our claim is established.

For the remainder of this paper let

$$
E=\left\{y \in L_{p \tau}^{2}[0,1]: y^{\prime} \in L_{p}^{2}[0,1] \text { and } y(1)=0\right\}
$$

For $u, v \in E$ we define

$$
\langle u, v\rangle=\int_{0}^{1} p \tau u \bar{v} d t+\int_{0}^{1} p u^{\prime} \overline{v^{\prime}} d t
$$


We show $E$ is complete. Let $\left\{y_{n}\right\}$ be a Cauchy sequence in $E$. Then there exist functions $y \in L_{p \tau}^{2}[0,1]$ and $u \in L_{p}^{2}[0,1]$ with $y_{n} \rightarrow y$ in $L_{p \tau}^{2}[0,1]$ and $y_{n}^{\prime} \rightarrow u$ in $L_{p}^{2}[0,1]$ as $n \rightarrow \infty$. Let

$$
v(t)=-\int_{t}^{1} u(s) d s
$$

Note $v(1)=0$. Also, notice since $y_{n} \in E\left(\right.$ so $\left.y_{n}(1)=0\right)$ that

$$
\begin{aligned}
\int_{0}^{1} p & (t) \tau(t)\left|y_{n}(t)-v(t)\right|^{2} d t \\
& =\int_{0}^{1} p(t) \tau(t)\left|\int_{t}^{1}\left(y_{n}-v\right)^{\prime}(s) d s\right|^{2} d t \\
& \leq\left(\int_{0}^{1} p(t) \tau(t) \int_{t}^{1} \frac{d s}{p(s)} d t\right)\left(\int_{0}^{1} p(s)\left|\left(y_{n}-v\right)^{\prime}(s)\right|^{2} d s\right) \\
& =\left(\int_{0}^{1} p(t) \tau(t) \int_{t}^{1} \frac{d s}{p(s)} d t\right)\left(\int_{0}^{1} p(s)\left|y_{n}^{\prime}(s)-u(s)\right|^{2} d s\right)
\end{aligned}
$$

and the right-hand side goes to zero as $n \rightarrow \infty$. Thus $y_{n} \rightarrow v$ in $L_{p \tau}^{2}[0,1]$ as $n \rightarrow \infty$, and so $y=v$ a.e. on $[0,1]$. As a result, $y_{n} \rightarrow v$ in $E$, so $E$ is complete. [In fact, in the following theorem, we could let $E$ be the space of functions $y \in L_{p \tau}^{2}[0,1]$ with $\left.y^{\prime} \in L_{p}^{2}[0,1].\right]$

Theorem 2.2. Suppose conditions (i) - (v) of Theorem 1.1 hold and assume problem $\left(P_{0}\right)$ has only the trivial solution. In addition, assume $f$ has the decomposition

$$
f(t, u)=g(t, u) u+h(t, u)
$$

where $g, h:[0,1] \times \mathbb{R} \rightarrow \mathbb{R}$ are Carathéodory functions satisfying conditions (iii) - (iv) of Theorem 2.1. Also, suppose the following conditions are satisfied:

(i) There exist $0 \leq-\tau_{1}, \tau_{2} \in C[0,1]$ with $\tau_{1}(t) \tau(t) \leq g_{1}(t, u) \leq \tau_{2}(t) \tau(t)$ for a.e. $t \in[0,1]$ and $u \in \mathbb{R}$.

(ii) $E=\Omega \oplus \Gamma$ where $\Omega \subseteq K^{\star}$ is finite-dimensional and for every $0 \neq y=u+v \in K^{\star}$ with $u \in \Omega, v \in \Gamma$ we have $R(y)>0$

where

$$
R(y)=\int_{0}^{1}\left[p\left(v^{\prime}\right)^{2}-\left(\tau-\tau \tau_{1}\right) p v^{2}\right] d t-\int_{0}^{1}\left[p\left(u^{\prime}\right)^{2}-\left(\tau-\tau \tau_{2}\right) p u^{2}\right] d t .
$$

Then problem $\left(P_{1}\right)$ has at least one solution.

Remark 2.1. The set $K^{\star}$ in condition (ii) here is as defined in condition (v) of Theorem 2.1. In (ii) we have $y=u+v$ with $u \in \Omega$ and $v \in \Gamma$, so $\int_{0}^{1} p \tau u v d t+\int_{0}^{1} p u^{\prime} v^{\prime} d t=$ 0 .

Proof of Theorem 2.2. We first show that there exists $\varepsilon>0$ with

$$
R(y) \geq \varepsilon\left(\|y\|_{p \tau}^{2}+\left\|y^{\prime}\right\|_{p}^{2}\right)
$$


for any $y \in K^{\star}$; here $y=u+v$ with $u \in \Omega$ and $v \in \Gamma$. If this is false, then there exists a sequence $\left\{y_{n}\right\} \subset K^{\star}$ with $\left\|y_{n}\right\|_{p \tau}^{2}+\left\|y_{n}^{\prime}\right\|_{p}^{2}=1$ and

$$
R\left(y_{n}\right) \rightarrow 0 \quad \text { as } n \rightarrow \infty .
$$

Note $y_{n}=u_{n}+v_{n}$ with $u_{n} \in \Omega$ and $v_{n} \in \Gamma$. Now there is a subsequence $S$ of integers with

$$
y_{n} \rightarrow y \text { in } L_{p \tau}^{2}[0,1] \quad \text { and } \quad y_{n}^{\prime} \rightarrow y^{\prime} \text { in } L_{p}^{2}[0,1]
$$

as $n \rightarrow \infty$ in $S$. Also, since strong and weak convergence are the same in finitedimensional spaces we have

$$
u_{n}^{\prime} \rightarrow u^{\prime} \quad \text { in } L_{p}^{2}[0,1] \text { as } n \rightarrow \infty \text { in } S .
$$

We also have

$$
\int_{0}^{1} p\left[v^{\prime}\right]^{2} d t \leq \liminf \int_{0}^{1} p\left[v_{n}^{\prime}\right]^{2} d t
$$

Now (2.11) - (2.14) imply that $R(y) \leq 0$. From assumption (ii) we have $y \equiv 0$. Finally (note $E=\Omega \oplus \Gamma$, so $\int_{0}^{1} p \tau u_{n} v_{n} d t+\int_{0}^{1} p u_{n}^{\prime} v_{n}^{\prime} d t=0$ ),

$$
\begin{aligned}
\left\|y_{n}\right\|_{p \tau}^{2}+\left\|y_{n}^{\prime}\right\|_{p}^{2}= & R\left(y_{n}\right)+\int_{0}^{1} p \tau\left[v_{n}^{2}+u_{n}^{2}\right] d t+2 \int_{0}^{1} p\left[u_{n}^{\prime}\right]^{2} d t \\
& +\int_{0}^{1}\left(\left[\tau-\tau_{1} \tau\right] p v_{n}^{2}-\left[\tau-\tau_{2} \tau\right] p u_{n}^{2}\right) d t \\
\rightarrow & 0 \text { as } n \rightarrow \infty \text { in } S
\end{aligned}
$$

which is impossible. Thus (2.10) holds for some $\varepsilon>0$.

Let $y(=u+v)$ be a solution to problem $\left(P_{\lambda}\right)$ for some $0<\lambda<1$. Then

$$
-\int_{0}^{1}(v-u)\left[\left(p y^{\prime}\right)^{\prime}+p \tau y\right] d t=-\lambda \int_{0}^{1} p(v-u) y g(t, y) d t-\lambda \int_{0}^{1} p(v-u) h(t, y) d t
$$

and so integration by parts yield

$$
\begin{gathered}
\int_{0}^{1}\left[p\left(v^{\prime}\right)^{2}+p v^{2}(-\tau+\lambda g(t, y))\right] d t-\int_{0}^{1}\left[p\left(u^{\prime}\right)^{2}+p u^{2}(-\tau+\lambda g(t, y))\right] d t \\
\leq \int_{0}^{1} p|v-u||h(t, y)| d t .
\end{gathered}
$$

Now

$$
\begin{aligned}
p v^{2}[-\tau+\lambda g(t, y)] & =p v^{2}\left[-\left(\tau-\tau_{1} \tau\right)+\lambda g(t, y)-\tau_{1} \tau\right] \\
& \geq p v^{2}\left[-\left(\tau-\tau_{1} \tau\right)+(\lambda-1) \tau_{1} \tau\right] \\
& \geq-p\left(\tau-\tau_{1} \tau\right) v^{2} \text { a.e. on }[0,1] .
\end{aligned}
$$

Similarly,

$$
p u^{2}[-\tau+\lambda g(t, y)] \leq-p\left(\tau-\tau_{2} \tau\right) u^{2} \quad \text { a.e. on }[0,1]
$$


Putting these into (2.15) yields

$$
R(y) \leq \int_{0}^{1} p|v-u \| h(t, y)| d t .
$$

This together with (2.10) implies that there is an $\varepsilon>0$ with

$$
\varepsilon\left(\|y\|_{p \tau}^{2}+\left\|y^{\prime}\right\|_{p}^{2}\right) \leq \int_{0}^{1} p|v-u||h(t, y)| d t .
$$

Next, notice that for $t \in(0,1)$ we have

$$
|v(1)-u(1)| \leq|v(t)-u(t)|+\int_{t}^{1}\left|(v-u)^{\prime}(s)\right| d s
$$

and so for $t \in(0,1)$

$$
|v(1)-u(1)| \leq|v(t)-u(t)|+\left\|v^{\prime}-u^{\prime}\right\|_{p}\left(\int_{t}^{1} \frac{d s}{p(s)}\right)^{\frac{1}{2}} .
$$

Note also that

$$
\|v-u\|_{p \tau}^{2}+\left\|v^{\prime}-u^{\prime}\right\|_{p}^{2}=\|y\|_{p \tau}^{2}+\left\|y^{\prime}\right\|_{p}^{2}
$$

and this together with (2.17) yields for $t \in(0,1)$

$$
|v(1)-u(1)| \leq|v(t)-u(t)|+\left(\|y\|_{p \tau}^{2}+\left\|y^{\prime}\right\|_{p}^{2}\right)^{\frac{1}{2}}\left(\int_{t}^{1} \frac{d s}{p(s)}\right)^{\frac{1}{2}} .
$$

Multiply this by $\sqrt{p(t) \tau(t)}$ and integrate from 0 to 1 (using Hölder's inequality) to obtain

$$
\begin{aligned}
\mid v(1) & -u(1) \mid \int_{0}^{1} \sqrt{p(t) \tau(t)} d t \\
& \leq\|v-u\|_{p \tau}+\left(\|y\|_{p \tau}^{2}+\left\|y^{\prime}\right\|_{p}^{2}\right)^{\frac{1}{2}}\left(\int_{0}^{1} p \tau \int_{t}^{1} \frac{d s}{p(s)} d t\right)^{\frac{1}{2}} .
\end{aligned}
$$

This together with (2.18) yields

$$
|v(1)-u(1)| \leq K_{2}\left(\|y\|_{p \tau}^{2}+\left\|y^{\prime}\right\|_{p}^{2}\right)^{\frac{1}{2}}
$$

where

$$
K_{2}=\frac{1+\left(\int_{0}^{1} p(t) \tau(t) \int_{t}^{1} \frac{d s}{p(s)} d t\right)^{\frac{1}{2}}}{\int_{0}^{1} \sqrt{p(t) \tau(t)} d t} .
$$

Also, for $t \in(0,1)$ we have

$$
|v(t)-u(t)| \leq|v(1)-u(1)|+\left\|v^{\prime}-u^{\prime}\right\|_{p}\left(\int_{t}^{1} \frac{d s}{p(s)}\right)^{\frac{1}{2}}
$$


and so (use (2.18) and (2.19)) for $t \in(0,1)$

$$
|v(t)-u(t)| \leq\left(\|y\|_{p \tau}^{2}+\left\|y^{\prime}\right\|_{p}^{2}\right)^{\frac{1}{2}}\left\{K_{2}+\left(\int_{t}^{1} \frac{d s}{p(s)}\right)^{\frac{1}{2}}\right\}
$$

In addition, since $y(1)=0$ we have $|y(t)| \leq \int_{t}^{1}\left|y^{\prime}(s)\right| d s$ for $t \in(0,1)$ and so

$$
|y(t)| \leq\left(\|y\|_{p \tau}^{2}+\left\|y^{\prime}\right\|_{p}^{2}\right)^{\frac{1}{2}}\left(\int_{t}^{1} \frac{d s}{p(s)}\right)^{\frac{1}{2}}
$$

for $t \in(0,1)$. Put condition (iii) of Theorem 2.1 into (2.16) to obtain

$$
\begin{aligned}
& \varepsilon\left(\|y\|_{p \tau}^{2}+\left\|y^{\prime}\right\|_{p}^{2}\right) \\
& \quad \leq \int_{0}^{1} p(t)|v(t)-u(t)| \phi_{1}(t) d t+\int_{0}^{1} p(t)|v(t)-u(t)||y(t)|^{\gamma} \phi_{2}(t) d t .
\end{aligned}
$$

This together with $(2.20)-(2.21)$ gives

$$
\begin{aligned}
\varepsilon\left(\|y\|_{p \tau}^{2}+\left\|y^{\prime}\right\|_{p}^{2}\right) \leq & \left(\|y\|_{p \tau}^{2}+\left\|y^{\prime}\right\|_{p}^{2}\right)^{\frac{1}{2}}\left[K_{2} \int_{0}^{1} p(t) \phi_{1}(t) d t+K_{0}\right] \\
& +\left(\|y\|_{p \tau}^{2}+\left\|y^{\prime}\right\|_{p}^{2}\right)^{\frac{\gamma+1}{2}}\left[K_{2} \int_{0}^{1} p(t) \phi_{2}(t)\left(\int_{t}^{1} \frac{d s}{p(s)}\right)^{\frac{\gamma}{2}} d t+K_{1}\right]
\end{aligned}
$$

where

$$
K_{0}=\int_{0}^{1} p(t) \phi_{1}(t)\left(\int_{t}^{1} \frac{d s}{p(s)}\right)^{\frac{1}{2}} d t \quad \text { and } \quad K_{1}=\int_{0}^{1} p(t) \phi_{2}(t)\left(\int_{t}^{1} \frac{d s}{p(s)}\right)^{\frac{\gamma+1}{2}} d t
$$

Now since $0 \leq \gamma<1$, there exists a constant $M>0$, independent of $\lambda$, with $\|y\|_{p \tau}^{2}+$ $\left\|y^{\prime}\right\|_{p} \leq M$. The result now follows from Theorem 1.1

\section{References}

[1] Chawla, M. M. and P. N. Shivakumar: On the existence of solutions of a class of singular nonlinear two point boundary value problems. J. Comp. Appl. Math. 19 (1987), 379 388.

[2] Dunninger, D. R. and J. C. Kurtz: A priori bounds and existence of positive solutions for singular nonlinear point boundary value problems. SIAM J. Math. Anal. 17 (1986), 595 $-609$.

[3] El Gebeily, M. A., Boumenir, A. and A. B. M. Elgindi: Existence and uniqueness of solutions of a class of two-point singular nonlinear boundary value problems. J. Comp. Appl. Math. 46 (1993), $345-355$. 
[4] Fonda, A. and J. Mawhin: Quadratic forms, weighted eigenfunctions and boundary value problems for nonlinear second order ordinary differential equations. Proc. Royal Soc. Edinburgh 112A (1989), 145 - 153.

[5] Mawhin, J. and W. Omano: Two point boundary value problems for nonlinear perturbations of some singular linear differential equations at resonance. Comm. Math. Univ. Carolinae 30 (1989), 537 - 550.

[6] O'Regan, D.: Nonresonant nonlinear singular problems in the limit circle case. J. Math. Anal. Appl. 197 (1996), 708 - 725.

[7] O'Regan, D.: Existence Theory for Nonlinear Ordinary Differential Equations. Dordrecht: Kluwer Acad. Publ. 1997.

Received 12.01.2001; in revised form 22.05.2001 